\title{
E-SSA Satellite Messaging Return Channel: Performance Analysis and Field Trials Results
}

\author{
T. Heyn ${ }^{*}$, P. Nagel ${ }^{*}$, B. Niemann*, A. Recchia ${ }^{\#}$, F. Collard ${ }^{\#}$, R. Hermenier ${ }^{\infty}$, M.A. Marchitti ${ }^{\infty}$, M. Andrenacci ${ }^{+}$, G. \\ Mendola \\ *Fraunhofer Institute for Integrated Circuits - IIS, Erlangen, Germany, \{thomas.heyn, peter.nagel, \\ bernhard.niemann\}@iis.fraunhofer.de \\ ${ }^{\#}$ Eutelsat, Paris, France, \{arecchia, fcollard\}@eutelsat.fr \\ ${ }^{\infty}$ DLR, Oberpfaffenhofen, Germany, \{romain.hermenier, maria-antonietta.marchitti\}@dlr.de \\ ${ }^{+}$MBI, Pisa, Italy, \{mandrenacci,gmendola\}@mbigroup.it
}

\begin{abstract}
An open ITS platform combining interactive satellite services with other communication channels is being developed and evaluated in field trials within the SafeTRIP project [1]. Prototyping and in-field validation of a novel waveform for messaging return channel over satellite for land mobile is an important objective of the project. The overall system architecture has recently been standardized by ETSI under the name of S-MIM (S-band Mobile Interactive Multimedia). The messaging protocol, described in the Part 3 of the standard, is based on the Enhanced Spread Spectrum Aloha (E-SSA). Its main asset resides in the low power required at the transmitter, which will allow the reuse of off-the-shelf power amplifiers and low-cost omnidirectional antennas.

This paper will present a comprehensive summary of previous E-SSA performance analysis from simulations and the first field trials results using the E-SSA waveform. The presented results have been derived from static and mobile field trials carried out in Germany with fully functional E-SSA modulator and demodulator prototypes and the EUTELSAT10A satellite.

Results for the static and mobile performance of the E-SSA demonstrator with an omni-directional antenna under Line-ofsight (LOS) conditions are presented. The measured Packet Error Rates of transmissions via satellite at different terminal power levels confirm the theoretical link budget calculations for single and multiple simultaneously transmitting terminals. The degradation due to fading effects of the transmission channel under mobile conditions has been measured during the trials to approx. $3 \mathrm{~dB}$. The resulting overall required transmitter power in the multi-user scenario of the trials setup has been only $-3 \mathrm{dBW}$ to reach a high QoS under mobile conditions. This value confirms the suitability of the E-SSA waveform for interactive mobile services for the mass market.
\end{abstract}

\section{Introduction. SATEllite Return ChanNel Systems}

The availability of the S-band frequencies for the development of Mobile Satellite Services (MSS) over the whole of Europe has opened the way to a truly innovative satellite communication system. Thanks to the S-band, the power consumption of the terminal is limited and the size of the antennas comparable to the commonly installed rooftop shark antennas. These features, in addition to the intrinsic "across-boundaries" nature of the satellite, make the S-band best suited to the new generation of "communicating cars". The main advantage of the system resides in the deployment of an ad-hoc messaging channel, allowing the interaction between the connected terminals and the satellite at affordable prices, thanks to the effectiveness of the ad-hoc return link employed protocol.

The overall architecture has recently been standardized by ETSI under the name of S-MIM (S-band Mobile Interactive Multimedia) [2]. The focus of this paper will be the Part 3 of the standard, which is based on the Enhanced Spread Spectrum Aloha (E-SSA) waveform, an asynchronous access protocol especially conceived to provide messaging services over the satellite return link. The absence of synchronization mechanisms simplifies deployment and activation of the terminals. A wide range of applications based on burst transmissions not significantly capacity-demanding have been envisaged, such as telemetry, environment and traffic monitoring, emergency alerts, fleet management, highway tolling, forecast predictions, pay-per-view. Road-safety and emergency applications have been tested and validated in a real scenario through the SafeTRIP demonstration platform. The validation of the applications is out of the scope of this paper. In the following chapters we will demonstrate the viability of the S-MIM transmission protocol for the return link messaging and we will show the on-field validation of the first standard-compliant prototype.

\section{E-SSA SYSTEM ARCHITECTURE}

In this section, a high level description of the lower layers of the E-SSA system architecture is given.

The protocol stack of the S-MIM return link asynchronous access is composed of three different planes: the User Plane, the Control Plane which supports and controls the User Plane functions, and the Management Plane.

The link layer of the User Plane is divided into four sublayers:

- Header Compression, which provides non-compressed as well as unidirectional stateless compressed channels to the higher layers; it is managed by the Header Compression Control.

- Transmission Mode, managed by the ARQ Management and Load Control function, providing three access services (a transparent mode and two acknowledged modes). 
- Return Link Encapsulation (RLE), used to encapsulate higher layer packets and to insert the system specific MAC layer addressing.

- Encryption, managed by the Link Layer Authentication, provides encrypted and non-encrypted channels.

At the physical layer there is a single transport channel, namely Random Access Channel (RACH) and two physical channels, namely Physical Data Channel (PDCH), to which the RACH is mapped, and Physical Control Channel (PCCH), used to carry physical layer signaling information.

More exhaustive insights of the E-SSA system architecture can be found in the standard [2] as well as in [3] and [4], which present overviews of the S-MIM specifications.

\section{E-SSA PERFORMANCE ANALYSIS FROM SIMULATIONS}

Simulations have been carried out in the framework of the DENISE project [5] so as to assess the performance of the ESSA radio interface in terms of aggregated throughput. [3] gives an overview of the overall DENISE system and provides first simulation results for this asynchronous access scheme. Considering a burst length of 1200 bits, the overall aggregate throughput can reach up to 5-6 Mbps for a bandwidth of $5 \mathrm{MHz}$ for different E-SSA terminals EIRP (the typical configuration for E-SSA radio interface adopted in DENISE can be found in [3]). Assuming that all the terminals transmit at the same data rate, namely $5 \mathrm{kbps}$ or $10 \mathrm{kbps}$, it corresponds respectively to 1200 or 600 parallel transmissions, considering one packet sent per user. Increasing the EIRP of E-SSA terminals does not necessarily lead to a higher overall aggregate throughput because of the high amount of interferences generated by the terminals: the E-SSA demodulator at the hub side can no longer decode the incoming packets [3].

Results, as depicted in Fig. 1 and Fig, 2, show that the aggregated throughput collapses when the offered traffic becomes too high, due to the increased level of interferences. The use of power randomization $\left(\mathrm{R}_{\max }\right.$ set to $3 \mathrm{~dB}$ in TABLE I) slightly increases the overall performance since in average the terminals EIRP are lower and thus less interference is generated. The major difference between scenarios S1 and S2 and scenarios S3 and S4 is the number of parallel transmissions which is the half in the scenarios S3 and S4 due to the higher individual data rate.

TABLE I

E-SSA SCENARIOS

\begin{tabular}{|l|c|c|c|c|}
\hline Scenario & S1 & S2 & S3 & S4 \\
\hline E-SSA terminal EIRP $[\mathrm{dBW}]$ & +2 & +2 & +5 & +5 \\
\hline Data Rate (in kbps) & 5 & 5 & 10 & 10 \\
\hline Spreading factor & 256 & 256 & 128 & 128 \\
\hline${\text { Power Randomization } \mathrm{R}_{\max }[\mathrm{dBW}]^{1}}^{1}$ & 0 & 3 & 0 & 3 \\
\hline
\end{tabular}

The previous study has been carried out for an E-SSA burst length of 1200 bits. In [3] and [4] two more burst sizes have been defined, namely 300 and 600 bits. The following

\footnotetext{
${ }^{1}$ Power randomization factor as explained in [6]
}

simulations have been performed considering the 600 bits burst length and using the environment defined in [6], where four scenarios have been edited (see TABLE I) in order to assess the performance of the E-SSA radio interface.

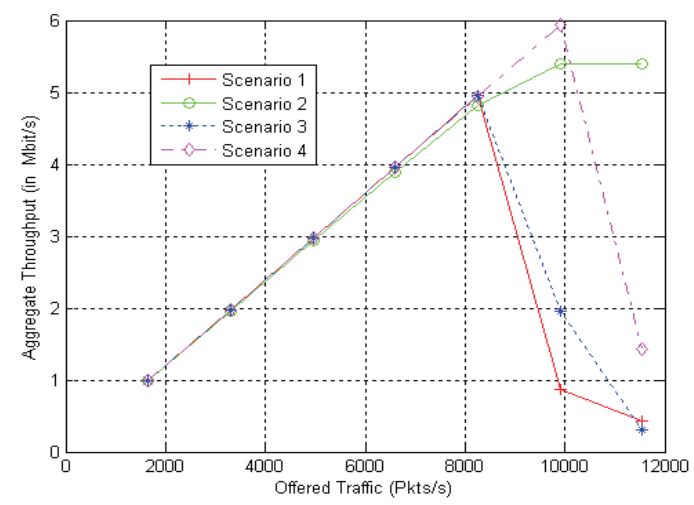

Fig. 1 Aggregated throughput considering 600 bits burst length

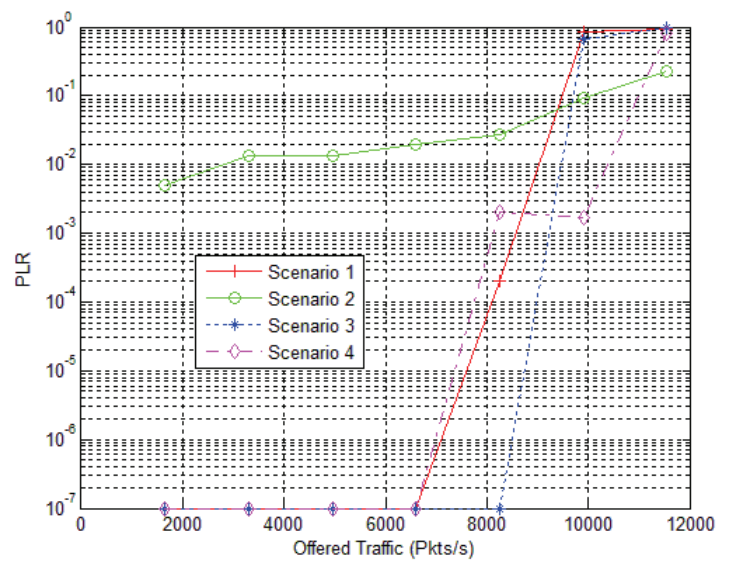

Fig. 2 Packet Loss Ratio (PLR) for the aggregated throughput considering 600 bits burst length

Focusing on a specific terminal EIRP and data rate (e.g. 5 dBW and $10 \mathrm{kbps}$ ), we show in Fig. 3 and Fig. 4 the obtained results merged with those from [3] for the two considered burst lengths (600 and 1200 bits). Hence, for a given aggregated throughput, the offered traffic load differs depending on the E-SSA burst length.

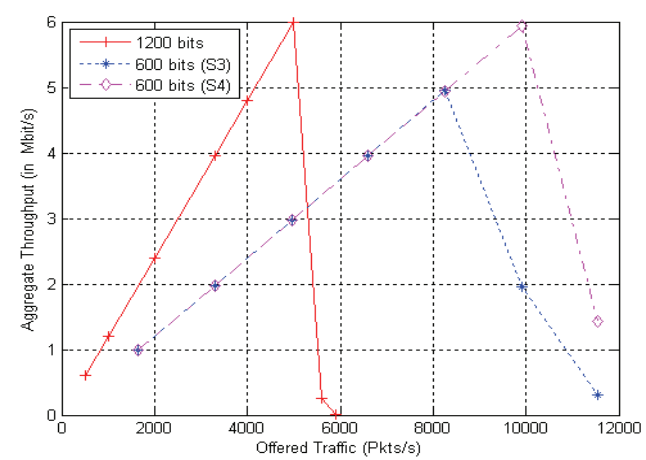

Fig. 3 Aggregated throughput of terminals with $5 \mathrm{dBW}$ EIRP for two different E-SSA burst lengths at $10 \mathrm{kbps}$ 


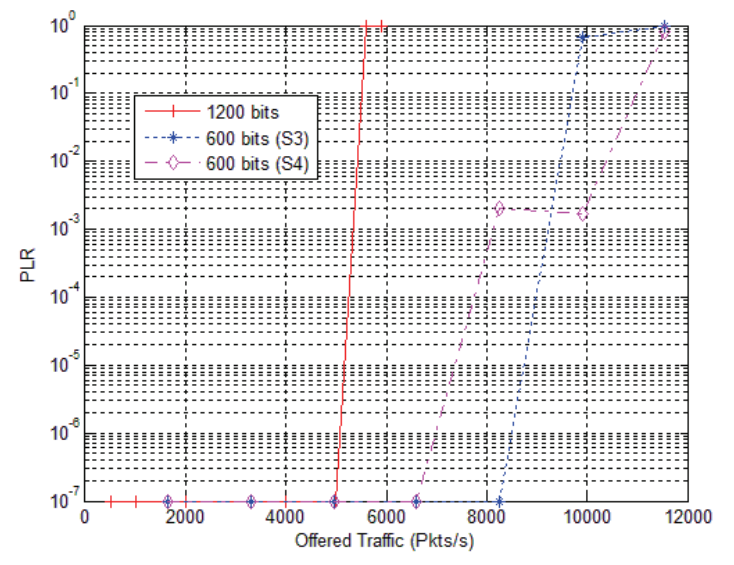

Fig. 4 PLR of terminals with 5 dBW EIRP for two different E-SSA burst lengths at $10 \mathrm{kbps}$

\section{FIELD MEASUREMENTS SETUP}

The early field trials within the SafeTRIP project focus on the verification of the theoretical link budget over the EUTELSAT10A satellite and the performance verification of the E-SSA implementation under static and mobile conditions.

A transmission system had been developed, comprising a mobile E-SSA modulator prototype and a demodulator, located in the Eutelsat satellite hub operation center in Rambouillet, France (Fig. 5). An additional component in the hub is the traffic emulator, which is simulating asynchronously transmitting terminals.

A prototype transmitter antenna with an omni-directional pattern has been selected for the trials, which is considered as typical for mobile terminals with small, non-directional carroof antennas. The passive antenna gain has been measured by Fraunhofer in the anechoic chamber and varies over the azimuth between +1 and $+3 \mathrm{dBic}$.

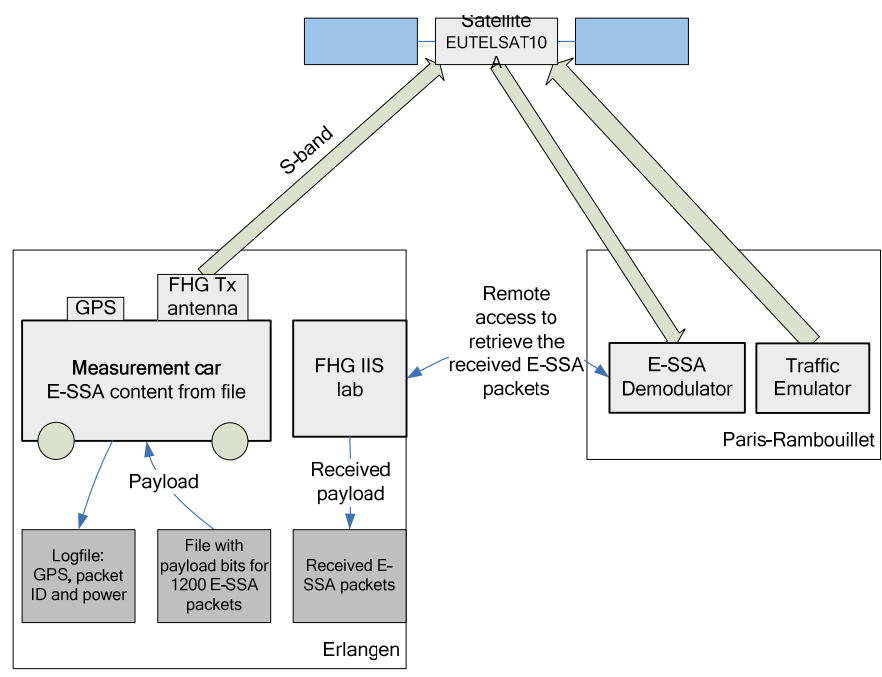

Fig. 5 Measurement setup for E-SSA trials

MBI has recently completed the implementation of the first prototype of an E-SSA demodulator, which is ETSI standard [2] compliant. The outcome of this activity is a software platform, called E-SSA test-bed, which can be used to demonstrate the S-band return channel both in a real scenario and in a laboratory.

The E-SSA test-bed is hosted on commercial hardware. The radio front-ends are implemented using the Software Defined Radio technology from Ettus corp. in order to provide the radio interface with a flexible frequency between $50 \mathrm{MHz}$ and $2.2 \mathrm{GHz}$. The computational power required to perform the fully software-based demodulation is achieved using commercial hardware equipped with the latest generation of GPUs. The elementary component of the E-SSA demodulator is called a node, comprising a server equipped with 4 GPUs. The nodes can be connected in cascade configuration in order to ensure hardware redundancy and the required throughput. On transmission side, the E-SSA test-bed is composed of a Traffic Emulator, which is able to generate an over-the-air composite signal, emulating a large population of S-MIM terminals, in addition to a channel emulator based on the Land Mobile Satellite (LMS) model from Perez-Fontan [7].

The E-SSA test-bed can be fully operated via web-based interfaces and includes a set of software modules for performance analysis. Finally, the E-SSA demodulator interfaces with the following communication stages using an open TCP/IP based protocol.

Both the traffic emulator and the E-SSA demodulator are installed at the S-band satellite hub in Paris-Rambouillet and are currently used in the framework of the SafeTRIP project together with the Fraunhofer S-MIM modulator to test the communication protocol over the EUTELSAT10A satellite.

The E-SSA modulator by Fraunhofer consists of both Hardand Software modules and is based on the Industrial PC Platform DT4900 (Fig. 6). The ESSA modulator comprises the following parts:

- Modulator SW running on the PC receiving Packets from the Link Layer.

- Modulator firmware running on an FPGA receiving turbo coded words from the PC transforming them into the digital transmit output signal.

- Modulator hardware that transforms the digital output of the FPGA into the analogue RF S-band signal to be provided to the power amplifier.

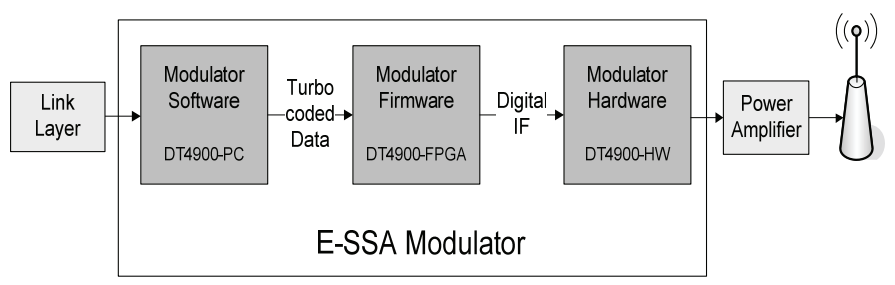

Fig. 6 Block diagram of E-SSA modulator prototype

The characteristics of the uplink signal from the terminal are shown in the Table II. A photo of the used antennas on the measurement car is depicted in Fig. 7. 
TABLE II

CHARACTERISTICS OF THE E-SSA UPLINK SIGNAL

\begin{tabular}{|c|c|}
\hline Parameter & Value \\
\hline Polarization & RHCP \\
\hline Centre frequency & $2,001.25 \mathrm{MHz}$ \\
\hline Signal band width & $2.34 \mathrm{MHz}$ \\
\hline Burst length & $240 \mathrm{~ms}$ \\
\hline Burst payload & 1200 bits \\
\hline Transmit power & $\begin{array}{c}\text { Depending on test between }-9.7 \text { and } \\
-2.7 \text { dBW EIRP (referred to the } \\
\text { average antenna gain) }\end{array}$ \\
\hline
\end{tabular}

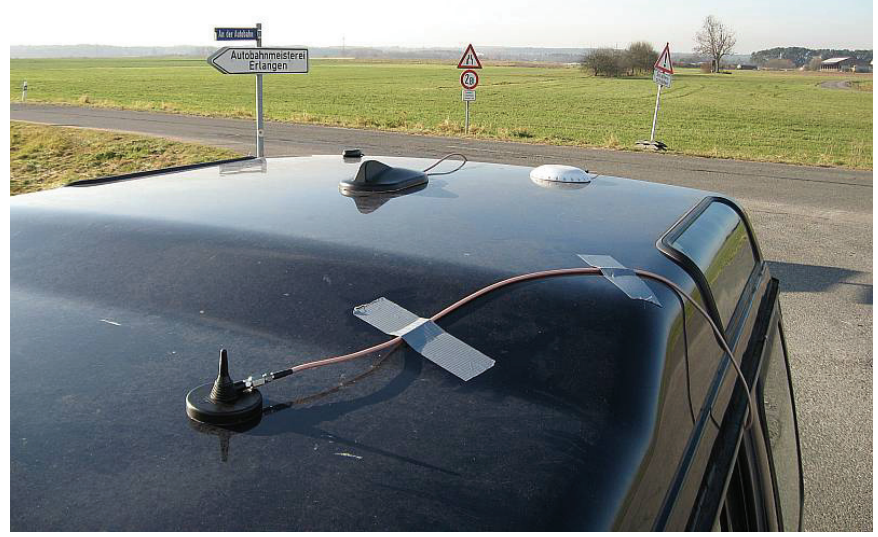

Fig. 7 Picture of mobile measurement setup (front: monitoring antenna for transmit spectrum; center: Fraunhofer S-band transmit antenna; white: DVB$\mathrm{SH}$ receiver antenna; left, back: GPS receiver)

\section{MeAsurement Results}

Static and mobile trials have been carried under the conditions as summarized in the following table:

TABLE III

MEASUREMENT CATEGORIES

\begin{tabular}{|c|c|c|}
\hline \multirow{2}{*}{ Mobility } & \multicolumn{2}{|c|}{ Traffic emulator state } \\
\cline { 2 - 3 } Static & On (multi-user scenario) & Off (single-user scenario) \\
\hline Mobile vs. transmit power, & QoS vs. transmit power, \\
& $\begin{array}{c}\text { link budget verification } \\
\text { link budget verification }\end{array}$ \\
\hline $\begin{array}{c}\text { performance (loss due to } \\
\text { fading channel) }\end{array}$ & $\begin{array}{c}\text { Characterization of } \\
\text { mobile performance (loss } \\
\text { due to fading channel) }\end{array}$ \\
\hline
\end{tabular}

\section{A. Static Link Budget Assessment: Theoretical Results and} on-field Validation

In order to validate the system, tests have been performed to match the link budget theoretical calculations with the onfield results.

The reference chain, hosted at Eutelsat premises in Rambouillet, includes the traffic generator and the demodulator (with one node during the trials). The overall RF chain is based on an uplink transmitting in S-band towards EUTELSAT10A satellite and a downlink platform receiving in Ku-band. A high power amplifier is inserted before the parabolic antenna in order to emulate an aggregated traffic up to several thousands of transmitting terminals per second and evaluate the performance at high MAC-load.

A probe, inserted at the output of the traffic generator and remotely controlled, allowed to monitor in real-time the power at the input of the antenna and, consequently, the transmitted EIRP towards EUTELSAT10A.

The first test has been done using a local loop between the modulator and the demodulator. A traffic uniformly distributed with a MAC-load of 4 packets/s has been generated and then an AWGN has been added, resulting in low $\mathrm{C} / \mathrm{N}$ values around $-25 \mathrm{~dB}$. The Power Density Function (PDF) issued by the demodulator clearly shows a step characteristic around a $\mathrm{C} / \mathrm{N}$ threshold value for $\mathrm{AWGN}$ channel.

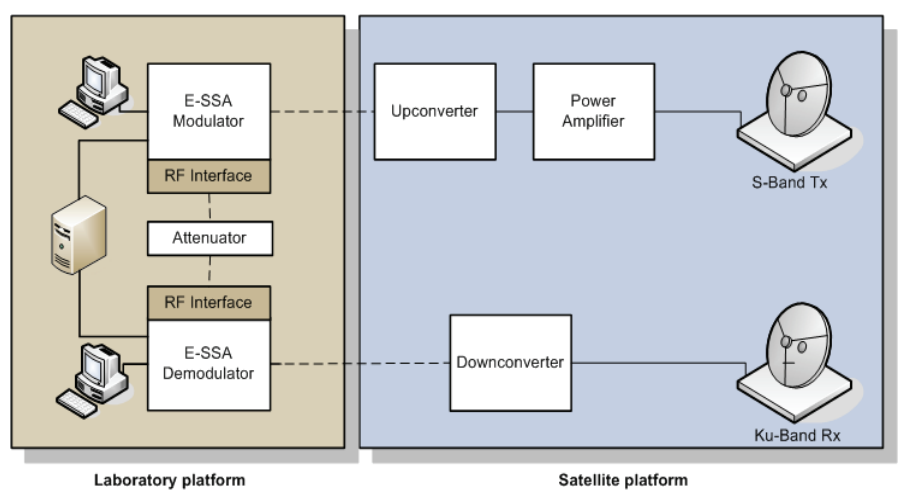

Fig. 8 Rambouillet platform

The same traffic has been transmitted via satellite as well, without adding additional noise. Knowing the emitted EIRP and the corresponding $\mathrm{C} / \mathrm{N}$ value, the demodulator threshold value has been found to be at $\mathrm{C} / \mathrm{N}=-25 \mathrm{~dB}$, when transmitting at very low MAC-loads.

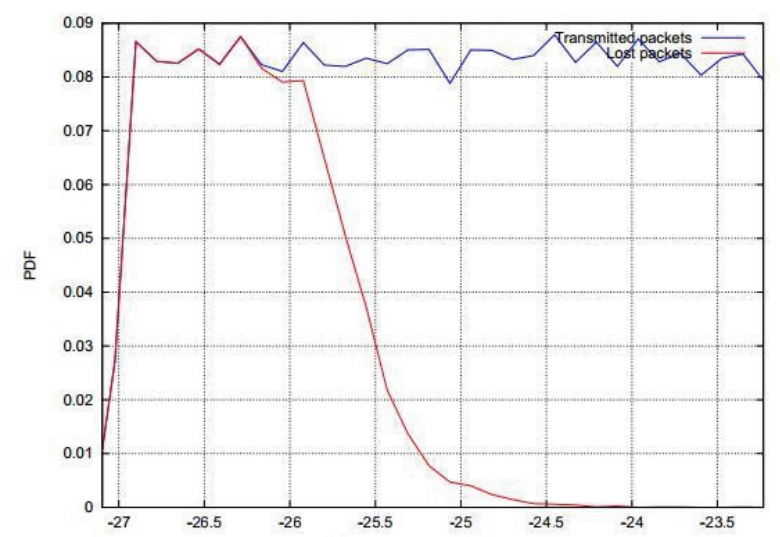

Fig. 9 PDF Distribution of transmitted and lost packets wrt. $\mathrm{C} / \mathrm{N}$ values

In the following tables, the link budgets calculated for the Fraunhofer terminal emitting from Erlangen and demodulation in Rambouillet are reported for single- and multi-user scenarios.

The demodulator threshold from the local loop perfectly copes with single-user scenario results experimentally found with the Fraunhofer modulator transmitting over satellite (Fig. 
9). In fact, the threshold measured during the trials in terms of transmitted power to reach a Packet Error Rate of $10^{-3}$ has been $-8.7 \mathrm{dBW}$ EIRP, which corresponds to a $\mathrm{C} / \mathrm{N}=-25 \mathrm{~dB}$.

In the multi-user scenario, the emulated traffic load has been chosen to simulate 500 packets/s (corresponding to 120 simultaneously transmitting terminals in average), which had been the limit of the demodulator installation in the hub during our trials. Under these conditions, the $\mathrm{E}_{\mathrm{b}} / \mathrm{N}_{\mathrm{t}}$ degradation due to interference between the terminals is 2.7 $\mathrm{dB}$ according to the theoretical link budgets. The settings of the traffic emulator have been the following: $1.92 \mathrm{Mchip} / \mathrm{s}$, 1200 bits of packet length, oversampling factor of 4, lognormal power distribution without clipping with a standard deviation $\sigma=3 \mathrm{~dB}$, no frequency drift has been added. The traffic has been generated using a Poisson distributed arrival process. The difference of the thresholds measured in the field under static conditions has been $3 \mathrm{~dB}$, which again confirms the theoretical link budget.

TABLE IV

THEORETICAL LiNK Budget (SINGLE TERMINAL)

\begin{tabular}{|c|c|}
\hline Parameter & Value \\
\hline Uplink frequency & $2,001.25 \mathrm{MHz}$ \\
\hline Occupied Bandwidth & $2.34 \mathrm{MHz}$ \\
\hline Chip-Rate & $1.92 \mathrm{Mcps}$ \\
\hline Burst length & $240 \mathrm{~ms}$ \\
\hline Code Rate & $1 / 3$ \\
\hline Terminal Location & Erlangen \\
\hline Latitude & $49.6^{\circ} \mathrm{N}$ \\
\hline Longitude & $11.01^{\circ} \mathrm{E}$ \\
\hline Terminal Tx Power & $-10 \mathrm{dBW}$ \\
\hline Cable loss & $1.7 \mathrm{~dB}$ \\
\hline $\begin{array}{l}\text { Antenna gain (at orientation } \\
\text { with highest gain) }\end{array}$ & $+3 \mathrm{dBic}$ \\
\hline Terminal EIRP & $-8.7 \mathrm{dBW}$ \\
\hline Spreading Loss & $162.7 \mathrm{~dB}\left(\mathrm{~m}^{2}\right)$ \\
\hline Free Space Loss & $190.12 \mathrm{~dB}$ \\
\hline IPDF & $-171.5 \mathrm{dBW} / \mathrm{m}^{2}$ \\
\hline $\mathrm{G} / \mathrm{T}$ & $9 \mathrm{~dB} / \mathrm{K}$ \\
\hline Received C/T & $-189.9 \mathrm{dBW} / \mathrm{K}$ \\
\hline Boltzmann constant & $-228.6 \mathrm{dBW} / \mathrm{Hz} / \mathrm{K}$ \\
\hline Uplink C/N & $-25 \mathrm{~dB}$ \\
\hline Downlink $\mathrm{C} / \mathrm{N}$ & $20.2 \mathrm{~dB}$ \\
\hline Final $\mathrm{C} / \mathrm{N}$ & $-25 \mathrm{~dB}$ \\
\hline $\mathrm{E}_{\mathrm{b}} / \mathrm{N}_{\mathrm{t}}$ & $1.7 \mathrm{~dB}$ \\
\hline
\end{tabular}

TABLE V

THEORETICAL Link Budget (Single Terminal + TRAFFiC EMUlator)

\begin{tabular}{|c|c|}
\hline Parameter & Value \\
\hline Terminal Location & Erlangen \\
\hline Terminal EIRP & $-8.7 \mathrm{dBW}$ \\
\hline $\begin{array}{c}\text { Emulated Terminals, } \\
\text { transmitting in parallel }\end{array}$ & $\begin{array}{c}120, \text { equivalent to } 500 \\
\text { packets } / \mathrm{s}\end{array}$ \\
\hline IPDF & $-144.6 \mathrm{dBW} / \mathrm{m}^{2}$ \\
\hline
\end{tabular}

\begin{tabular}{|c|c|}
\hline Received C/T & $-163.1 \mathrm{dBW} / \mathrm{K}$ \\
\hline Uplink C/N (1 terminal) & $-25 \mathrm{~dB}$ \\
\hline Uplink C/N (total) & $-4.2 \mathrm{~dB}$ \\
\hline Downlink C/N & $20.2 \mathrm{~dB}$ \\
\hline Final C/N $(1$ terminal $)$ & $-25 \mathrm{~dB}$ \\
\hline Final C/N (total) & $-4.2 \mathrm{~dB}$ \\
\hline $\mathrm{E}_{\mathrm{b}} / \mathrm{N}_{\mathrm{t}}(1$ terminal $)$ & $-1 \mathrm{~dB}$ \\
\hline
\end{tabular}

\section{B. Results from Mobile Trials}

Purpose of the mobile trials has been the performance analysis of the E-SSA transmission system under mobile Lineof-Sight (LOS) conditions. The Packet Error Rate (PER) has been measured for the transmissions of the measurement car in Erlangen with and w/o the traffic emulator signal.

The route had a length of approx. $3 \mathrm{~km}$ in an open environment w/o trees. As a consequence, the PER can be determined easily w/o the need to consider obstacles along the route. Since the closed route comprises all possible driving directions from 0 to $360^{\circ}$, an average antenna gain of $2 \mathrm{dBic}$ is assumed in the results for the terminal EIRP.

The difference between stationary and mobile transmissions has been calculated in order to determine the required transmit power of the terminals. The following two figures show the calculated Packet Error Rate (PER) for single terminal (Fig. 10) and multi-user scenario (Fig. 11). The required terminal EIRP to reach a PER of $10^{-3}$ are $-5.7 \mathrm{dBW}$ and slightly above $-2.7 \mathrm{dBW}$ in a single- and multi-user scenario respectively. The PER results with enabled traffic emulator are not as steep as for a single terminal, which might be caused by the adaptive decoding threshold of the demodulator as well as the extra noise coming from the power flux density of the emulated traffic. However, a high QoS of $99.6 \%$ successfully decoded packets can be reached in the mobile multi-user scenario for a terminal power of $-2.7 \mathrm{dBW}$ EIRP.

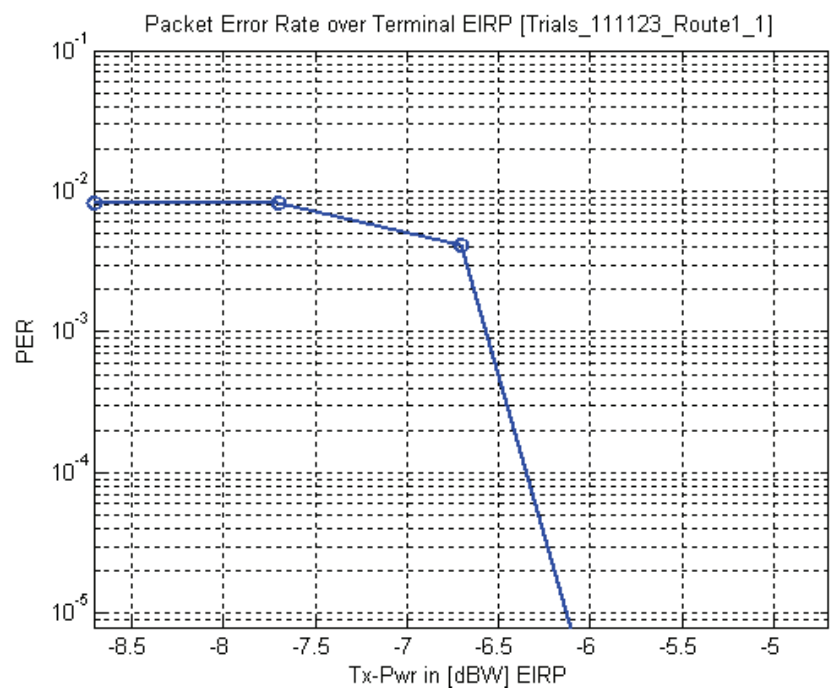

Fig. 10 PER vs. EIRP for mobile transmissions, with traffic emulator switched off. Minimum EIRP is $-5.7 \mathrm{dBW}$ (referred to average antenna gain). 


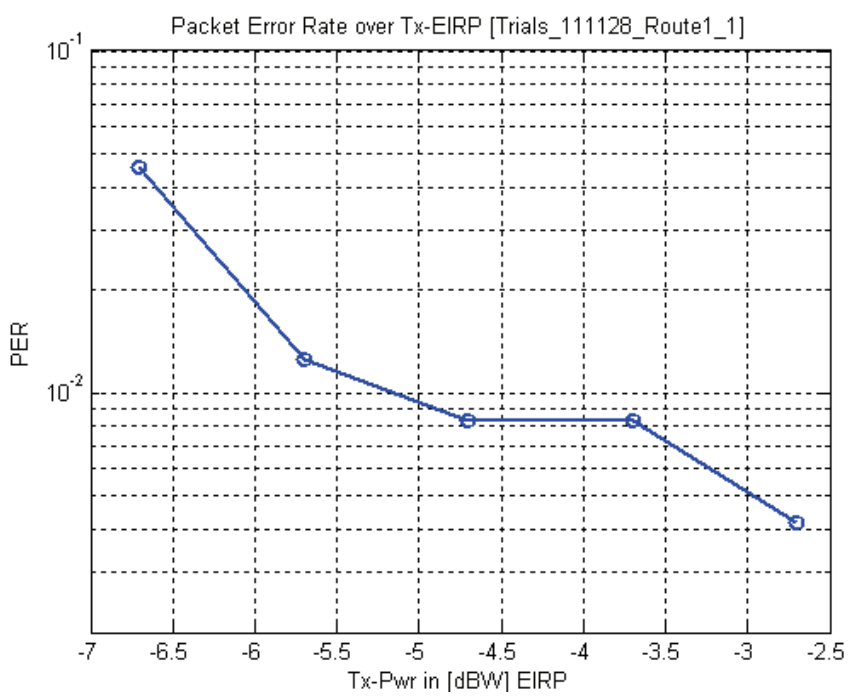

Fig. 11 PER vs. terminal EIRP (referred to average antenna gain) for mobile transmissions, with traffic emulator switched on.

The required thresholds for static and mobile transmissions are summarized in the table below. To summarize, the additional margin in the terminal for the mobile fading channel is approx. $3 \mathrm{~dB}$. The required terminal power during the trials for the multi-user scenario with 120 parallel transmitting mobile users is approx. $-2.7 \mathrm{dBW}$ EIRP. It has to be noted, that our test setup can be considered as a typical configuration of a commercial terminal with an omnidirectional antenna.

The difference between the observed transmitter power during the trials and the power of the terminal classes as specified in the DENISE project [5] is caused by two effects: Firstly, the initial assumption for the link budget in DENISE have been worst case transmission parameters and different to those of the used satellite EUTELSAT10A. The second reason is the number of simultaneous transmitting users in the multi-user scenario. The required terminal transmitter power in a scenario to reach the maximum E-SSA system throughput is subject for further studies and trials.

TABLE VI

REQUIRED TERMINAL POWER UNDER DIFFERENT CONDITIONS

\begin{tabular}{|c|c|c|}
\hline Required terminal EIRP & $\begin{array}{c}\text { Single user } \\
\text { scenario }\end{array}$ & $\begin{array}{c}\text { Multi-user } \\
\text { scenario }\end{array}$ \\
\hline $\begin{array}{c}\text { Static, } \\
\text { (antenna gain is }+3 \mathrm{dBic} \text { in } \\
\text { best position) }\end{array}$ & $\begin{array}{c}-8.7 \mathrm{dBW} \\
(\mathrm{PER}=0)\end{array}$ & $\begin{array}{c}-5.7 \mathrm{dBW} \\
\left(\mathrm{PER}=10^{-3}\right)\end{array}$ \\
\hline $\begin{array}{c}\text { Mobile, } \\
(\text { antenna gain }+1 \text { to }+3 \mathrm{dBic})\end{array}$ & $\begin{array}{c}-5.7 \mathrm{dBW} \\
(\mathrm{PER}=0)\end{array}$ & $\begin{array}{l}-2.7 \mathrm{dBW} \\
\left(\mathrm{PER}=4^{*} 10^{-3}\right)\end{array}$ \\
\hline
\end{tabular}

\section{CONCLUSIONS}

The results presented throughout this paper confirm the effectiveness of the S-MIM messaging protocol based on E-SSA. The main asset of the system resides in the low power required at the transmitter side, which will allow the reuse of off-the-shelf low-cost power amplifiers and omnidirectional antennas.
Additional performance tests are conducted within the SafeTRIP project in laboratory with the E-SSA HW setup to evaluate the achievable performance of the messaging system. Further trials with the SafeTRIP OBU, supporting all applications as described in [1], will be carried out until autumn 2012; results will be available by the end of the year.

\section{ACKNOWLEDGMENT}

The authors of this paper wish to thank the partners within the SafeTRIP consortium. They are in particular thankful to the FP7 and the European Commission for funding the SafeTRIP project.

\section{REFERENCES}

[1] SAFETRIP Project webpage: $h$ ttp://www safetrip.eu

[2] ETSI TS 102 721, "Satellite Earth Stations and Systems (SES); Air Interface for S-band Mobile Interactive Multimedia (S-MIM)", V1.1.1

[3] Scalise, Sandro and Párraga Niebla, Cristina and Gallinaro, Gennaro and Andrenacci, Marco and Rinaldo, Rita and Del Rio Herrero, Oscar and Breiling, Marco and Finocchiaro, Daniele and Cebrian Puyuelo, Joan Manuel and Schlüter, Gerald (2010) System Design for PanEuropean MSS Services in S-Band. In: IEEE Xplore Digital Library. 5th Advanced Satellite Multimedia Systems Conference, 13.-15. Sep. 2010, Cagliari, Italien.

[4] Scalise, Sandro and Párraga Niebla, Cristina and Arcidiacono, Antonio and Finocchiaro, Daniele and De Gaudenzi, Riccardo and Del Rio Herrero, Oscar and Schlüter, Gerald and Krause Jens (2012) S-MIM: a Novel Radio Interface for Efficient Messaging Services over Satellite. Workshop on Telecommunications: From Research to Standards, IEEE ICC 2012.

[5] DENISE Project webpage: http://telecom.esa.int/DENISE

[6] Hermenier, Romain and Scalise, Sandro (2011) Performance Assessment of an S-band Return Link for Messaging and Mobile Interactive Broadcasting. In: Proceedings of 17 th $\mathrm{Ka}$ and Broadband Communications, Navigation and Earth Observation Conference 2011, pp. 513-520. $2011 \mathrm{Ka}$ and Broadband Communications, Navigation and Earth Observation Conference, 3-5 Oct 2011, Palermo, Italy.

[7] Perez-Fontan et al., "Statistical Modeling of the LMS Channel", IEEE Transactions on vehicular technology, Vol. 50, No. 6, November 2001 